Research Article

\title{
Coupled Analysis of the Film-Cooling and Infrared Characteristics of an Axisymmetric Vectored Nozzle
}

\author{
Xiaoying Zhang and Rui Li \\ South China University of Technology, Wushan Road, Guangzhou 510641, China \\ Correspondence should be addressed to Xiaoying Zhang; zxy1119@scut.edu.cn
}

Received 7 January 2015; Accepted 23 March 2015

Academic Editor: Sotirios Karellas

Copyright ( 2015 X. Zhang and R. Li. This is an open access article distributed under the Creative Commons Attribution License, which permits unrestricted use, distribution, and reproduction in any medium, provided the original work is properly cited.

\begin{abstract}
This study adopts a narrow band model to investigate the cooling and radiation of a vectoring nozzle and to compute the gas spectral characteristic in infrared band. The radiative heat transfer between the hot gas and the wall is considered with an enclosure model. The calculation of film cooling is performed through a cooling effectiveness method. A coupled heat balance equation of heat flux and wall temperature is established on the multilayer structure of the nozzle, including the wall, heat shield, and outer shield, and a Newton-Raphson scheme is taken for solution. Temperature on the expansion part of an experimental nozzle in NASA TN D-1988 is investigated for verification. Another vectoring nozzle with a multirow of film cooling is also investigated. This study shows that the film in the heat shield remarkably cools the convergent part of the nozzle, thereby increasing the temperature on the expansion part of the nozzle. The deflection of the nozzle can change the distribution of the wall temperature and the radiation on the expansion part, which is lower on the deflection side than on the opposite side. The radiation from the nozzle outlet is high, particularly along the deflection direction in the rear hemisphere.
\end{abstract}

\section{Introduction}

Research on high-performance aeroengines shows that the nozzle is always the most severely heated structure of the engine. The gas temperature of a vectoring nozzle under afterburning condition is higher than $2000 \mathrm{~K}$ [1]. To prevent heating damage on the nozzle wall, film cooling has been widely applied on the vectoring nozzle in the past decades. The infrared radiation characteristics of a deflection nozzle are important for the engine to achieve infrared stealth and radar tracking [2]. In the vectoring nozzle of the aeroengine, computation of the wall temperature is a complex nonlinear problem that involves multiple coupled variables, including gas radiation and wall temperature. The major radiative gases, $\mathrm{H}_{2} \mathrm{O}$ and $\mathrm{CO}_{2}$, are all strongly spectral dependent, and their infrared radiation is composed of millions of spectral lines.

The waveband model of gas spectral radiation is generally computation intensive. The deflection of the nozzle breaks the axisymmetric distribution of gas flowing parameters and radiation, which also increases the difficulty and calculation of heat transfer analysis. Thus, the accurate coupled calculation of the film-cooling wall temperature and gas infrared radiation is a problem that must be solved in the heat protection optimization of a high-performance aeroengine.

The technology of applying film cooling on the convergent part of a nozzle was first studied on a rocket engine. Reference [3] demonstrated that when the pressure of a combustion chamber is higher than $5000 \mathrm{lbs} / \mathrm{in}$, only $40 \%$ of the wall heat flux can be removed by regenerative cooling; the use of film cooling to enhance cooling also proposed. Reference [4] proposed the Hatch-Papell equation to calculate film cooling in a rocket engine and used empirical methods to calculate the radiative heat. Reference [5] experimentally researched the influence of the geometry of the film hole on the film-cooling heat transfer coefficient. Reference [6] studied a combustion chamber with a wiggle strip film-cooling construction using empirical correlations of gas emissivity to compute the wall temperature distribution. Reference [7] focused on the wall temperature distribution of multiple slots of film cooling on heat shields to account for the heat conduction at the conjunction surface by using gas emissivity to compute radiative heat flux. Reference [8] numerically studied the mixing of the film and main flows on a plane with a single-film slot. Reference [9] researched the coupled 
computation methods of flow and the heat transfer of gas, wall, and coolant flow in a thrust chamber of a high-thrust liquid rocket engine. Reference [10] adopted the commercial software FLUENT to simulate the flow field and wall temperature of a round to square nozzle. Reference [11] researched the heat transfer model of an axisymmetric divergent nozzle and considered the conductive, convective, and radiative heat. Lefebvre empirical methods were adopted to compute the gas radiation to the wall.

Based on the spectral characteristics of aeroengine gas radiation, [12] adopted a weighted-sum-of-gray-gas model to simulate gas radiation; it used finite volume methods to compute the radiation transport equation. Reference [13] developed a $2 \mathrm{D}$ simulation model of radiation that uses a spherical harmonic method of solving the directed integral form of radiance equation. Given the complexity of gas spectral radiation calculation, few published studies have considered gas spectral radiance in analyzing the engine wall temperature. The cooling of new high-performance aeroengines and infrared stealth technology require the accurate prediction of the radiative heat transfer between the nozzle wall and gas. The present work established a theoretical analysis model that focuses on the entire structure of an aeroengine axisymmetric vectoring exhaust nozzle (axisymmetric vectored nozzle) and the convective/radiative heat transfer of hot gas. The temperature distribution of the entire nozzle structure, including heat shield, nozzle wall, and outer shield, is computed, as well as the infrared radiation on the wall and outlet surface of the nozzle.

\section{Geometric Model and Grid}

The vectoring nozzle with a multirow of film cooling investigated in this work is a $0.624 \mathrm{~m}$ long axisymmetric nozzle with a $0.8 \mathrm{~m}$ diameter afterburning part. The convergent part of the nozzle is $0.184 \mathrm{~m}$ long. The heat shield is set on the nozzle, with a gap of $0.006 \mathrm{~m}$ from the wall. Three slots of film cooling are applied on the heat shield. The coolant flows through the slots and covers the heat shield surface, thereby forming the adherent film. A part of the coolant directly goes through the end of the gap, rushes to the divergent part of the nozzle, and quickly mixes with the main flow. The outer shield outside the nozzle has a space of $0.1 \mathrm{~m}$ from the nozzle wall. The secondary flow goes through the space and conveys some of the heat on outside surface of the nozzle wall. The entire structure of the nozzle is shown in Figure 1. The diagram of the three slot of film is shown in Figure 2, where the three slots are equally spaced along length of the heat shield. Height of each slot is $1.5 \mathrm{~mm}$.

The calculation of the wall radiation heat and film-cooling heat transfer in this work is based on the flow field results of the computational fluid dynamics (CFD) software FLUENT. The CFD solution adopts an unstructured grid. We have done a series of calculations based on different grid resolution to get the grid-independent solution. The final grid resolution we adopted is about 107550 grids on $x-y$ coordinate plane, spaced $0.008 \mathrm{~m}$ apart in nozzle axis. The near zone $y+$ value is about $30 \sim 90$.

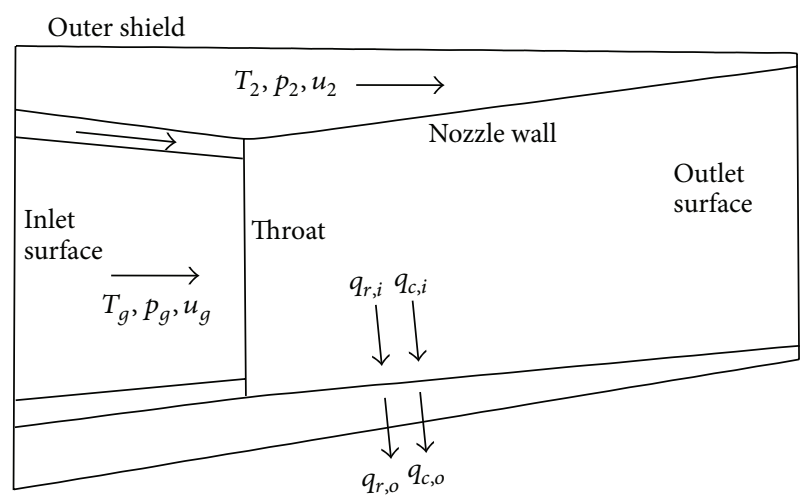

FIgURE 1: Geometric model of a vectoring nozzle.

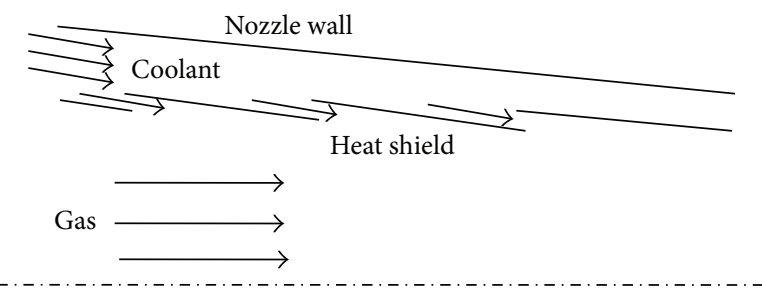

FIgURE 2: Diagram of three slots film cooling.

The grids system for CFD solution is not suitable for heat transfer and wall temperature computation, because of the irregular and too dense arrangement of nodes, particularly at the boundary layer of the wall. The density of grid nodes slows down the calculation of gas radiation. Therefore, we reset the grids for radiation calculation. The rearranged grids are divided with the equal space idea on 51 sections. The arrangement of the nodes in each section is shown in Figure 3. The gas properties at each node are obtained through a spatial interpolation method. The new set grids are quadrangular and regular, which facilitates the calculation of heat transfer. The final reset grids for computing heat transfer and wall temperature of the nozzle are shown in Figure 4.

\section{Calculation Methods}

3.1. Calculation Model of Gas Radiation [14]. An enclosure radiation model is used to calculate the hot gas radiation to the wall. An enclosure is established; it consists of the included hot gases, nozzle wall, inlet surface, and outlet surface. The radiation of each wall unit of the enclosure is calculated. The net radiative heat transfer rate of each unit is given as follows:

$$
q_{r}=\frac{\varepsilon_{w}}{1-\varepsilon_{w}}\left(\sigma T_{w}^{4}-J\right)
$$

where $\varepsilon_{w}$ is the emissivity of each unit. $T_{w}$ is the temperature of the wall unit. $J$ is the radiosity of each unit. The radiosity of 


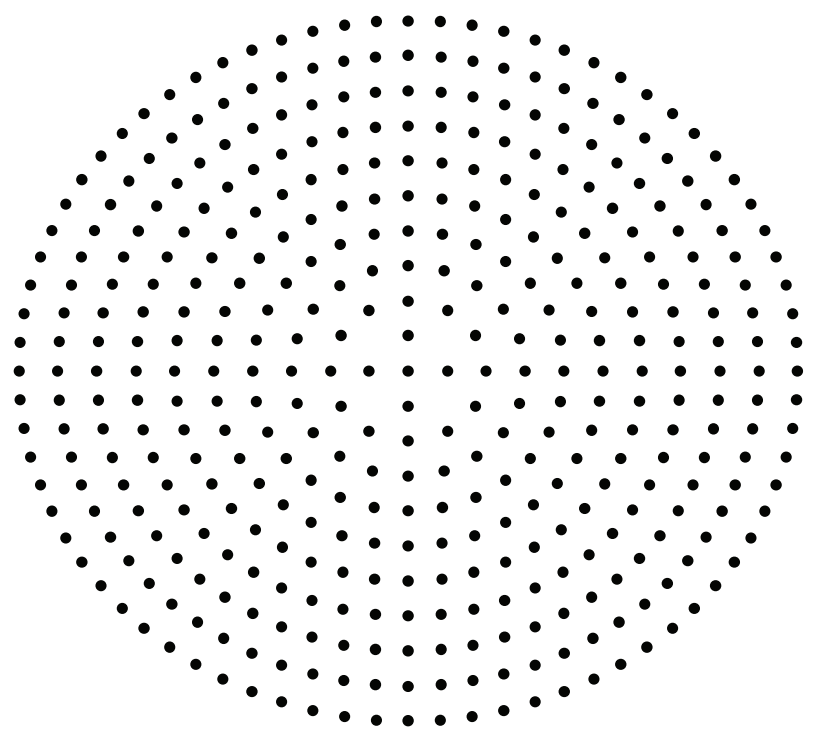

FIGURE 3: Grid nodes on a cross section.

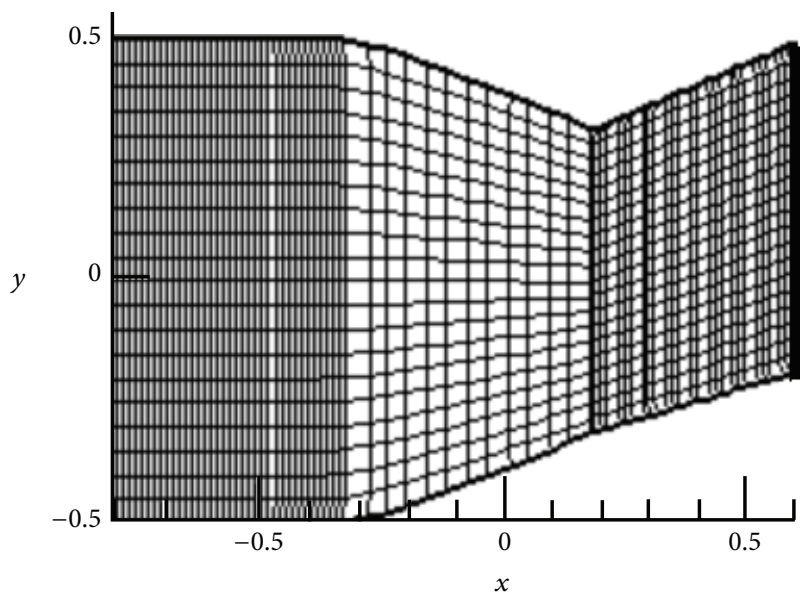

FIGURE 4: Grids for heat transfer and wall temperature computation.

unit $J$ consists of two parts: the radiation from the unit itself $E$ and the reflection of incident radiation $G$. Thus,

$$
J=E+\rho G,
$$

where $\rho$ is the wall reflectivity. Incident radiation $G$ comes from radiosity of all the other wall cells in the enclosure. Therefore, the radiosity of all wall cells should be correlated with one another and must be solved simultaneously.

On any of the wall cells, the incident radiation $G$ consists of the gas radiation of the incident hemisphere and the radiation from the other wall cells. To calculate $G$, the integral of the incident radiation in each direction of the hemisphere is needed. The incident hemisphere of a wall unit $\Delta A_{i}$ is divided into several solid angles $d \omega_{i}$.

Each solid angle corresponds to a unit surface $\Delta A_{k}$ on the wall. The incident radiation of each solid angle is calculated.
Their sum is used to obtain the total incident radiation of a wall unit. The incident radiation of each solid angle consists of gas radiation along the path and radiosity from the emitting wall unit $\Delta A_{k}$.

The incident radiation at each solid angle is the integral of the gas layer on the incident path, expressed as follows:

$$
\begin{aligned}
i_{\lambda, k}= & {\left[J_{\lambda, k} \tau_{\lambda, k}\right.} \\
& \left.+\sum_{n_{g}}\left\{e_{\lambda b}\left(\kappa_{\lambda}^{*}\right) \cdot\left\{\tau\left[\kappa_{\lambda}-\left(\kappa_{\lambda}^{*}+\delta \kappa_{\lambda}^{*}\right)\right]-\tau\left[\kappa_{\lambda}-\kappa_{\lambda}^{*}\right]\right\}\right\}\right] \\
& \cdot \frac{\cos \theta_{k} \cos \theta_{i} \Delta A_{k}}{\pi s^{2}}
\end{aligned}
$$

where $i_{\lambda, \kappa}$ represents the incident radiation along the connection of wall cells $\Delta A_{i}$ and $\Delta A_{k}$. The symbol $\kappa_{\lambda}$ is the optical thickness between the two wall cells, $\kappa_{\lambda}^{*}$ is the optical thickness from one specific calculation node to the incident wall, and $\delta \kappa_{\lambda}^{*}$ is the optical thickness within each calculation gas layer. $e_{\lambda b}\left(\kappa_{\lambda}^{*}\right)$ represents the black body radiation at the gas temperature. $\tau\left(\kappa_{\lambda}\right)$ represents the gas transmittance of optical thickness $\kappa_{\lambda} \cdot n_{g}$ is the gas layer number on the connection of the two wall cells (i.e., the number of gas layers in radiation integration). $J_{\lambda, k} \tau_{\lambda, k}$ indicates the part of the radiosity from the wall unit $\Delta A_{k}$ that goes through the intermediate gas to the wall unit $\Delta A_{i}$.

Considering the spectrum of gas radiation, the infrared radiation at any direction at the spectrum from $1 \mu \mathrm{m}$ to $5 \mu \mathrm{m}$ is divided into 41 discrete wavebands. Gas radiation in all the other spectrums is regarded as a transparent body. Thus, 42 groups of spectral radiosity $J_{\lambda, k}$, can be integrated to give radiosity in all wavelength.

For the other waveband outside the infrared waveband, a simplified equation ignoring the gas radiation is given as follows:

$$
i_{\lambda, k}=J_{\lambda, k} \frac{\cos \theta_{k} \cos \theta_{i} \Delta A_{k}}{\pi s^{2}} .
$$

In each waveband, the integral of (3) in all the solid angles of the hemisphere of $\Delta A_{i}$ is substituted into (2). Assume that the radiation angle factor is $X_{k i}=\cos \theta_{k} \cos \theta_{i} \Delta A_{k} / \pi s^{2}$, so the radiosity of one wall cell $\Delta A_{i}$ can be given as follows:

$$
\begin{aligned}
& J_{\lambda, i} \\
& =\varepsilon_{w} e_{\lambda b}+\rho_{w} \sum_{n_{k}} i_{\lambda, k} \\
& =\varepsilon_{w} e_{\lambda b}^{i}
\end{aligned}
$$




$$
\begin{aligned}
+\rho_{w} \sum_{n_{k}}\{[ & J_{\lambda, k} \tau_{\lambda, k} \\
& +\sum_{n_{g}}\left\{e_{\lambda b}\left(\kappa_{\lambda}^{*}\right)\right. \\
& \left.\left.\cdot\left\{\tau\left[\kappa_{\lambda}-\left(\kappa_{\lambda}^{*}+\delta \kappa_{\lambda}^{*}\right)\right]-\tau\left[\kappa_{\lambda}-\kappa_{\lambda}^{*}\right]\right\}\right\}\right] \\
\left.\cdot X_{k i}\right\} &
\end{aligned}
$$

where $n_{k}$ is the number of the infinitesimal solid angles in the incident hemisphere of the wall cell $\Delta A_{i}$. The radiosity equations of all the wall cells in the enclosure are a closed set of equations:

$$
\begin{gathered}
J_{\lambda, 1}=\varepsilon_{w} e_{\lambda b, 1}+\rho_{w} \sum_{n_{k}} i_{\lambda, k i}, \\
J_{\lambda, 2}=\varepsilon_{w} e_{\lambda b, 2}+\rho_{w} \sum_{n_{k}} i_{\lambda, k i}, \\
\vdots \\
J_{\lambda, n}=\varepsilon_{w} e_{\lambda b, n}+\rho_{w} \sum_{n_{k}} i_{\lambda, k i} .
\end{gathered}
$$

The coefficient matrix of this equation is diagonal dominant and can be solved using the Gauss-Seidel method. The radiosity of all the wall cells in every waveband is obtained, and their sum is the total radiosity. Thus, the relation between the net radiation heat $q_{r}$ and wall temperature $T_{w}$ is obtained according to (1).

3.2. Calculation Model of Film Cooling. A cooling effectiveness model is adopted to calculate the film cooling on the convergent part of the nozzle. The convective heat transfer rate $q_{c}$ of gas is computed according to the imperial correlation given by $[14,15]$.

The convective heat transfer rate of film cooling is given as follows:

$$
q_{c}=h_{c}\left(T_{w}-T_{a w}\right)
$$

where $T_{a w}$ is the adiabatic wall temperature of film cooling, $T_{w}$ is the temperature of the nozzle wall, and $h_{c}$ is the convective heat transfer coefficient. The common solution to $h_{c}$ is given by the forced convection heat transfer DittusBoelter formula. The physical parameter used in the formula is the main flow parameter near the wall. $T_{a w}$ is given by the empirical correlation of the cooling effectiveness. Consider

$$
\eta_{a w}=\frac{T_{g}-T_{a w}}{T_{g}-T_{\text {film }}}
$$

where $T_{g}$ is the temperature of mail flow in gas.
In this work, three cooling slots are applied on the heat shield. To calculate the compound cooling effectiveness of the three slots, the cooling effectiveness of multiple slots is calculated based on the result of a single slot. The cooling effectiveness equation of a single slot is given in [14] as follows:

$$
\eta=\eta_{0} \beta, \quad \beta=\frac{1+0.05 x / M S}{1+0.1 x / M S},
$$

where $M=\rho_{\text {film }} u_{\text {film }} / \rho_{g} u_{g}$ is the film blowing ratio, $x$ is the distance to the slot, $S$ is the slot height, and $\eta_{0}$ is function of $M$ and $x / S$. When the calculation point is close to the injection slot, the cooling effectiveness given by (10) is greater than 1, which is unreasonable. Under this condition, the Hatch-Papell equation is used instead, as recommended by [4]. In [4], the Hatch-Papell equation was applied on a rocket engine's remaining high precision. The Hatch-Papell equation is rewritten in metric form as follows:

$$
\begin{aligned}
\ln \eta= & -\left(\frac{3.6 \alpha_{i} p x}{g_{\text {film }} c_{p, \text { film }}}-K\right)\left(\frac{S u_{g}}{a_{\text {film }}}\right)^{1 / 8} f\left(\frac{u_{g}}{u_{\text {film }}}\right) \\
& +\ln \cos 0.8 \beta_{\text {eff. }}
\end{aligned}
$$

The compound cooling effectiveness of the three slots of film is given by [14] as follows:

$$
\eta=1-\left(1-\eta_{1}\right)\left(1-\eta_{2}\right)\left(1-\eta_{3}\right)
$$

where $\eta_{1}, \eta_{2}$, and $\eta_{3}$ are the cooling effectiveness of each slot.

3.3. Calculation Model of Wall Temperature. Lumped parameter methods are adopted to calculate the distribution of the wall temperature of all the nozzle structures. A heat balance equation of convective and radiative heat transfer is established on every wall unit as follows:

$$
q_{w}=q_{c, i}+q_{r, i}+q_{c, o}+q_{r, o}=0
$$

where the subscript $c$ represents the convective heat, $r$ means the radiative heat, $i$ means the inner side of the wall, and $o$ means the outer side of the wall.

The calculation of the convective and radiative heat of the wall cells whose inner side is directly heated by gas is discussed in the section above. The convective heat of other wall cells is calculated using the Dittus-Boelter equation. The radiative heat is calculated using the following equation of two envelope surfaces:

$$
q_{r, o}=\frac{\sigma\left(T_{w 1}^{4}-T_{w 2}^{4}\right)}{1 / \varepsilon_{1}+1 / \varepsilon_{2}-1} .
$$

The convective terms of the heat balance equation of the wall cells not directly heated by gas can also be solved using the Dittus-Boelter equation.

Considering any wall cell, the radiative term computed with (13) in its heat balance equation involves the temperature of the other wall cells. Thus, these heat balance equations are coupled with one another. Assuming $T_{w 1}, T_{w 2}$, and $T_{w 3}$ are the temperatures of heat shield, nozzle wall, and outer shield, 
respectively, $Q_{1}, Q_{2}$, and $Q_{3}$ are their heat balance equations; then the coupled heat balance equations group of the three structures above can be given as follows:

$$
\begin{gathered}
Q_{1}\left(T_{w 1}, T_{w 2}\right)=0, \\
Q_{2}\left(T_{w 1}, T_{w 2}, T_{w 3}\right)=0, \\
Q_{3}\left(T_{w 2}, T_{w 3}\right)=0 .
\end{gathered}
$$

Equation (14) is a set of multivariate nonlinear equations. These equations can be established on every wall cell of a nozzle. These equations are solved using the NewtonRaphson methods in our program, which performs iteration in each coupled equation.

In the iteration of (14), the radiative term of the gas-heated wall correlates with its radiosity and temperature. Also the radiosity of a wall cell correlates with the temperature of the other wall cells. Hence, the calculation of the radiosity and temperature of the wall cells should be simultaneous. When the temperature of a wall unit changes, iteration of radiosity must be repeated to update the radiosity of all wall cells. However, the waveband model for gas radiation consumes considerable time to complete the radiosity computation. Thus, the real-time coupling of temperature and radiosity is difficult to achieve.

The solution we adopt is stepwise and round coupling. First, a set of initial temperatures of the wall cells is assumed. According to these initial temperatures, the heat balance equation is iterated, ignoring the gas radiation. A set of wall temperatures is obtained. Second, the iteration of radiosity is performed according to the wall temperature above. The gas radiative heat transfer rate of the wall cells is generated. Thirdly, according to the newly generated radiative heat, the iteration of the wall temperature is restarted. A set of wall temperatures that consider the gas radiation is obtained and regarded as the result of first round. A new round of iteration will be restarted if temperature difference between two rounds is greater than a minimum limit. Iteration errors of both heat balance and gas radiation get smaller in a new round of iteration.

\section{Results and Discussion}

4.1. Results and Analysis of the Verification Case. To verify theoretical and numerical model of this work, a film-cooling aeroengine [6] and a rocket engine [4] are investigated. Reference [6] measured the temperature distribution from the fourth to the end of the sixth wiggle strip of a flame tube of Spey-25 engine. The flame tube of this engine used film cooling on the wiggle strip. The result of this work is compared with that of [4] in Figure 5, where nondimensional temperature is plotted against nondimensional distance. Nondimensional temperature is the ratio of the temperature of the calculation point and the maximum temperature. Nondimensional distance is the ratio of the axial distance to the inlet and the nozzle length. Figure 5 shows that the results of the reference and this work reflect the cooling effect of film cooling. The wall temperature near the injection point is low and increases downstream, where three waves are found near

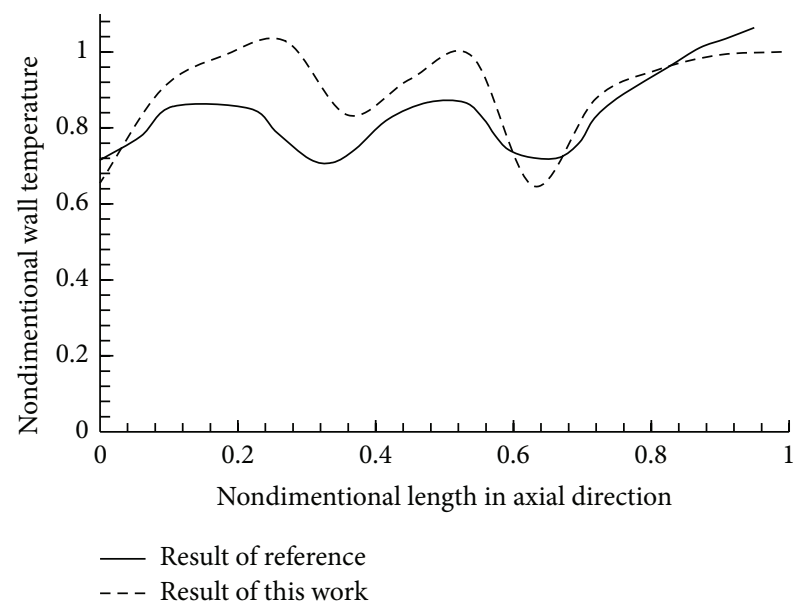

Figure 5: Comparison with [4].

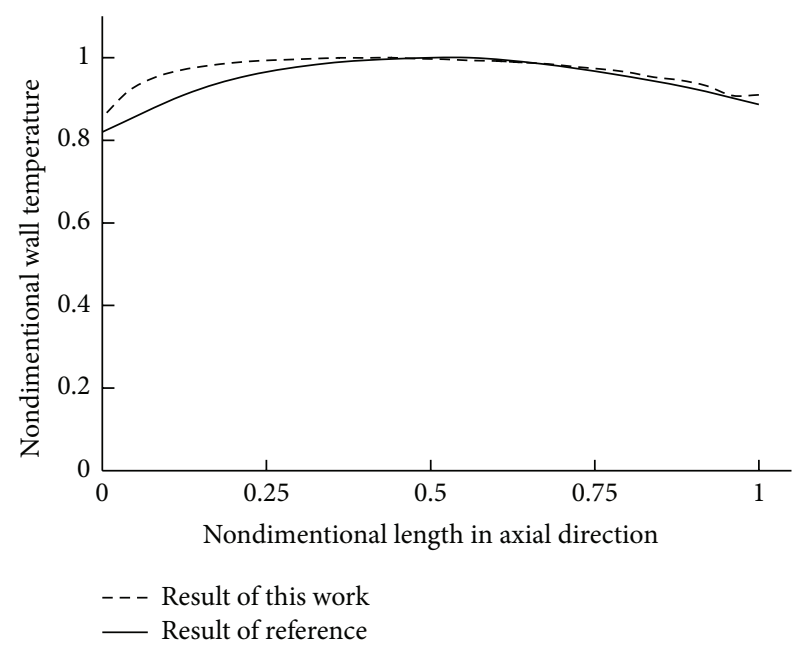

FIGURE 6: Comparison with [6].

three slots. Cooling effect of slot film is the strongest just at the injecting position, which will be gradually attenuated with distance increasing, as coolant will be heated in mixing with the gas. A new rise of cooling effect will come where a new slot appears.

The measured curve is more gradual in the said reference than in this work. The conductive heat transfer inside the wall is ignored in this work to simplify the calculation. However, high-temperature wall cells transfer heat to the neighboring low-temperature cells, thereby resulting in a gradual distribution of temperature. The difference between the results of the reference and this work is no more than $20 \%$. Therefore, the result of this work can properly reflect the variation trend of the wall temperature under film cooling.

Reference [4] gives the measured temperature distribution of a rocket engine under ground test condition. One slot of film cooling was applied on a slight upstream of the nozzle throat. A comparison of this work and the wall temperature $30 \mathrm{~s}$ after ignition in [6] is shown in Figure 6. The two groups of results basically agree with each other. However, the result 


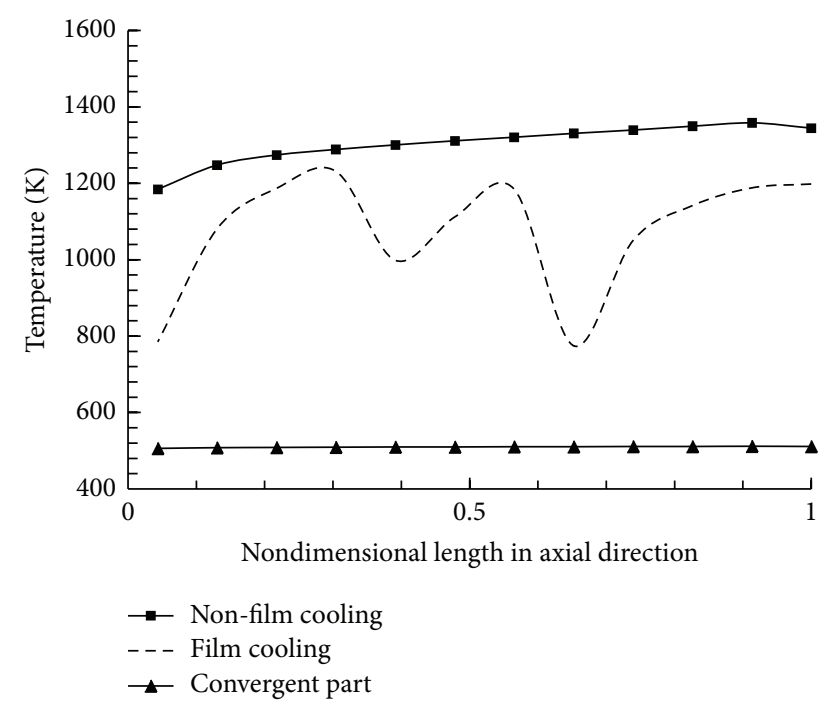

FIgURE 7: Wall temperature of the heat shield and convergent part.

of this work has a maximum temperature that is closer to the throat. The axisymmetric vectored nozzle in this work is deflected after the throat, thus causing temperature to ascend after the throat because of the scour of hot gas.

\subsection{Temperature Distribution of Axisymmetric Vectored Noz-}

zle. For the vectoring nozzle described in Section 2, this work calculates the temperature of its convergent part, as shown in Figure 4. The definition of nondimensional distance is the same as above. The temperature distribution of nonfilm cooling is given in Figure 7 for an intuitive comparison. Under the protection of film cooling, the wall temperature is significantly low. The fluctuation of wall temperature becomes great after applying the cooling film. The wall temperature near the coolant injection point can be $550 \mathrm{~K}$ lower when no film is applied. Under the isolation of the heat shield, the temperature of the convergent part of the nozzle is maintained at nearly $500 \mathrm{~K}$, which is approximately $500 \mathrm{~K}$ lower than that of the heat shield. While it is cooled by the film coolant in the gap of the heat shield, the nozzle wall does not have direct contact with the gas. The heat shield, accordingly, plays a good part in cooling the nozzle wall.

The temperature distribution on each structure of the nozzle is shown in Figure 8. The divergent part is more severely heated than the other parts of the nozzle. Without the protection of the heat shield, this part of the nozzle is directly exposed to the hot gas. Although the gas temperature after the nozzle obviously decreases, the wall temperature ascends to 940 K. Meanwhile, the average temperature of the outer shield increases from $440 \mathrm{~K}$ to $690 \mathrm{~K}$.

The main effects of vectoring condition on temperature distribution are the uneven distribution of gas parameters after the throat and the occlusion of the throat to the upper wall cells after the throat. The wall temperature distribution of vectoring condition in three circumferential positions is shown in Figure 9, where $\varphi$ is the circumferential angle to the deflection direction. The temperature of the deflection

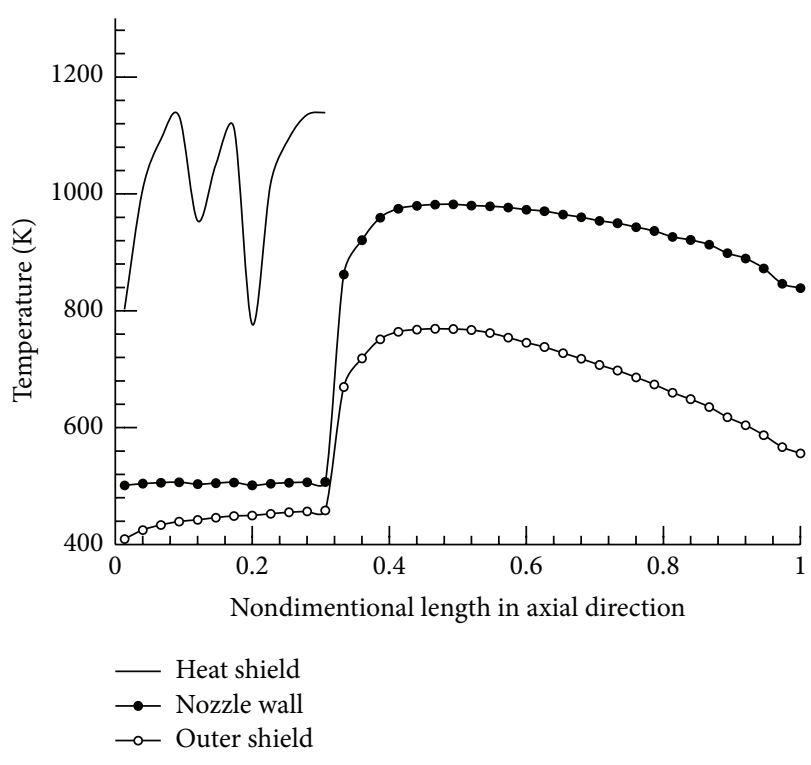

FIgURE 8: Temperature of the entire nozzle structure.

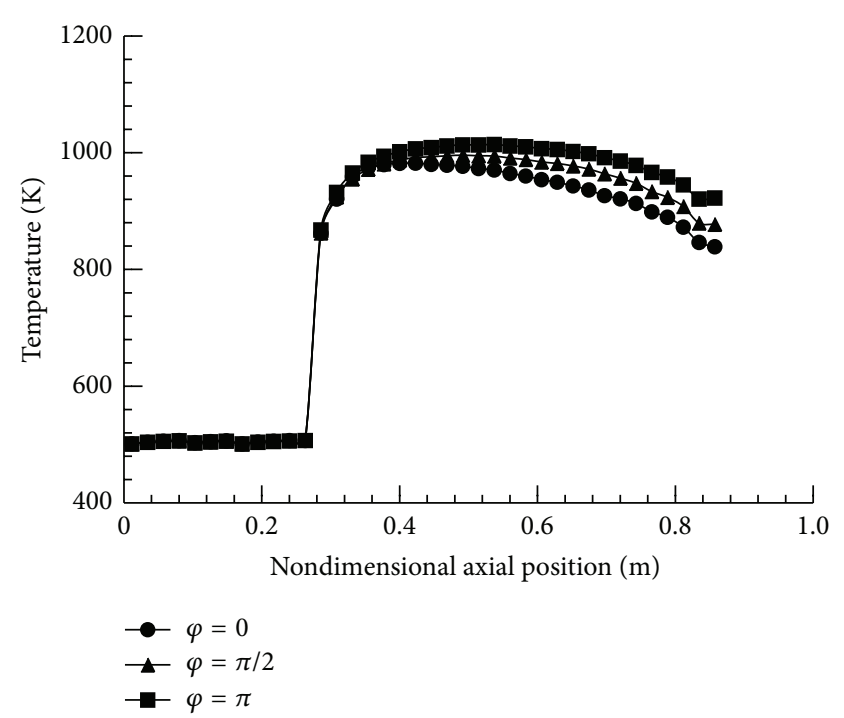

FIGURE 9: Temperature distribution at different circumferential angles.

direction $(\varphi=0)$ is the lowest, whereas that of the opposite direction $(\varphi=\pi)$ is the highest. The opposite direction is directly heated by gas. This part of the wall cells is not blocked by the throat and is heated directly by the inlet surface radiation. The average wall temperatures of these two directions differ by $4.8 \%$.

4.3. Comparison of Deflection and Nondeflection Conditions. The wall temperature distribution of the nondeflection condition is also obtained in this work. Compared with the deflection of the $20^{\circ}$ condition, as shown in Figure 10, the temperature distribution pattern at the divergent part (circumferential $\varphi=0$ ) may be different. The temperature curve of the deflection condition is arched because of the scour of 


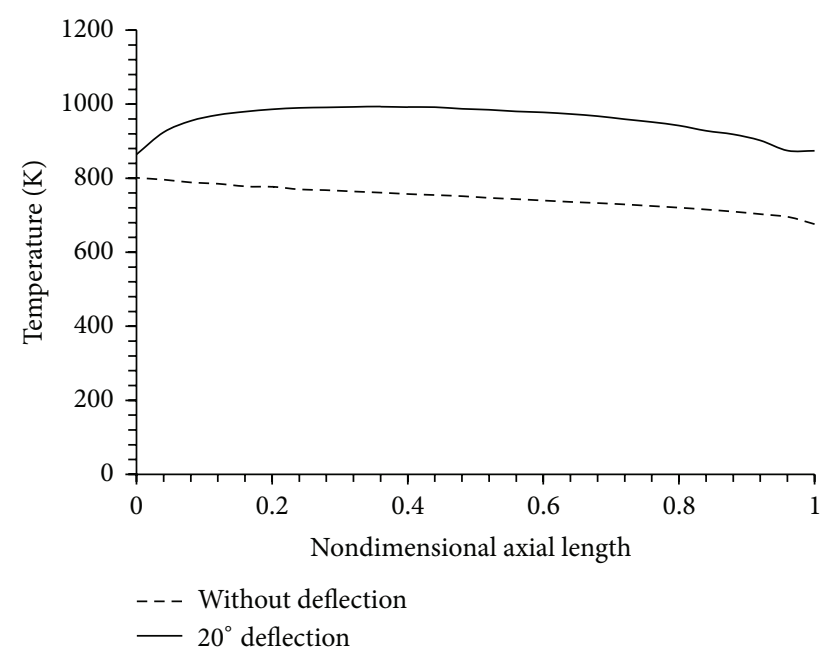

FIgURE 10: Comparison of $20^{\circ}$ deflection and nondeflection conditions.

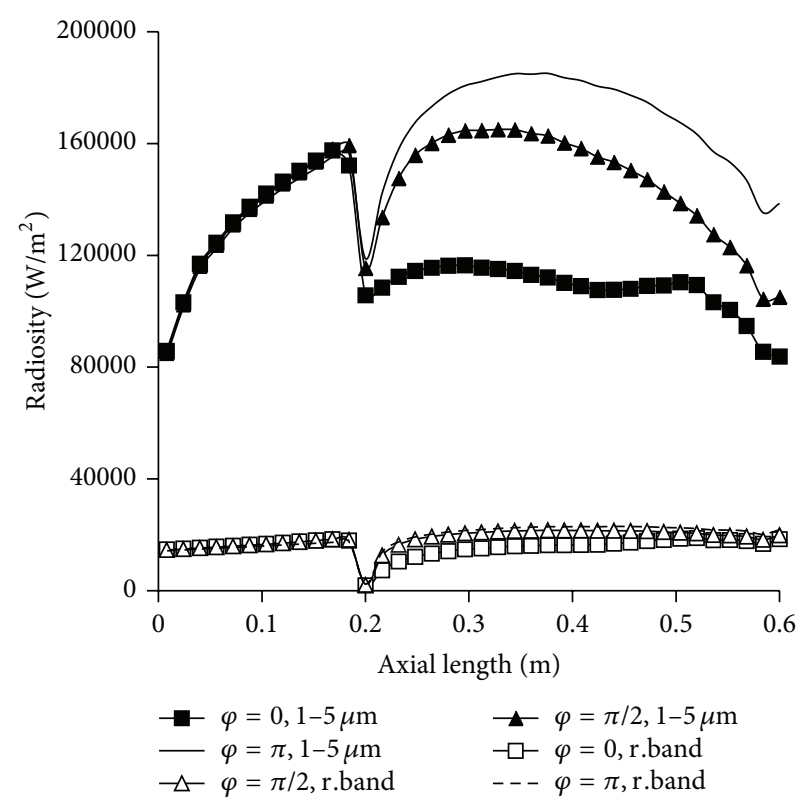

FIGURE 11: Radiosity in infrared and other wavebands on the wall.

hot gas, whereas the curve of the nondeflection condition exhibits a linear decline. The decline of the nondeflection condition is fast, and the maximum value appears at the throat. By contrast, the wall temperature of the deflection condition is maintained at a high value at the downstream of the throat, and the maximum temperature appears at a distance from the throat.

4.4. Infrared Characteristics of Axisymmetric Vectored Nozzle. Figure 11 shows the radiosity at an infrared waveband of $1 \mu \mathrm{m}$ to $5 \mu \mathrm{m}$ and other wavebands in the circumferential angle of $\varphi=0, \pi / 2$, and $\pi$ under vectoring condition. The radiosity at a waveband of $1 \mu \mathrm{m}$ to $5 \mu \mathrm{m}$ is 10 times greater than radiosity

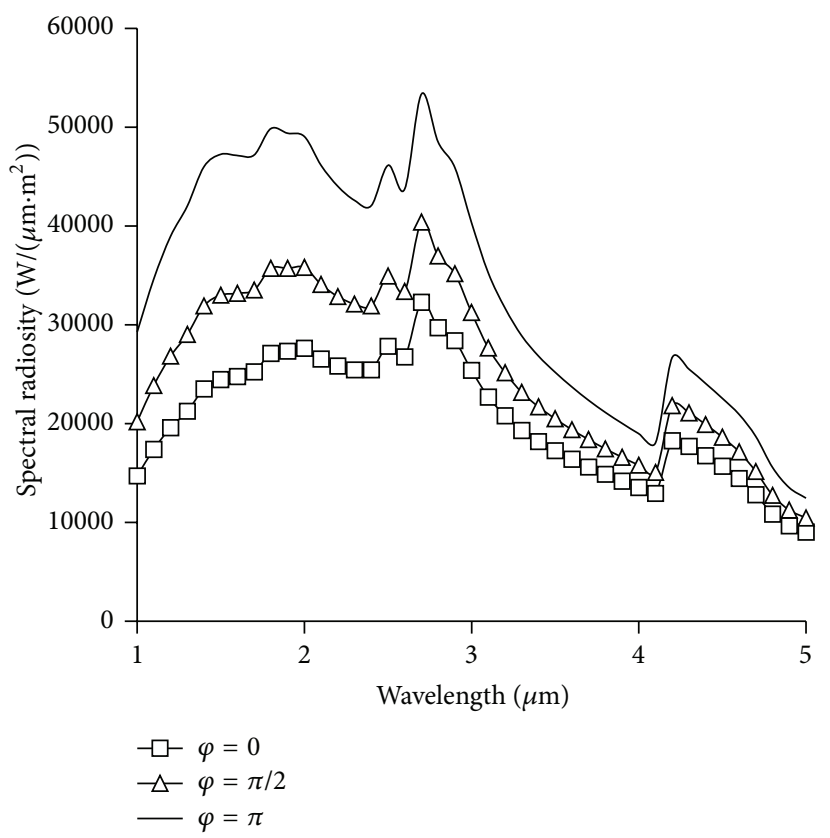

FIGURE 12: Spectral radiosity on the outlet surface.

at other wavebands. Therefore, the radiation of the nozzle is mainly concentrated in the infrared waveband. The radiosity in the convergent part is circumferentially uniform, whereas that in the divergent part has a minimal value when $\varphi=0$. The maximum value appears at $\varphi=\pi$. The radiosity at the throat is lower than that on the wall on both sides because of the contraction of the flow area and the deflection of the nozzle, which block the radiation of nearby gas of the other side. The nearby gas has the most significant effect on the wall, thereby decreasing gas radiation near the throat.

Figure 12 shows the spectral radiation at an infrared waveband of $1 \mu \mathrm{m}$ to $5 \mu \mathrm{m}$ on the outlet surface of the divergent part in the $\varphi=0, \pi / 2$, and $\pi$ direction. The spectral radiation on the outlet surface shows a characteristic of gas spectral radiation. When the spectral peak of $\mathrm{H}_{2} \mathrm{O}$ and $\mathrm{CO}_{2}$ radiation at $1.8,2.7$, and $4.3 \mu \mathrm{m}$ is reached, the spectral radiosity is enhanced. The circumferential difference of the radiosity has the same pattern as that of the wall temperature (i.e., the minimum value appears at $\varphi=0$, and the maximum value appears at $\varphi=\pi$ ).

4.5. Radiation Intensity at the Outlet. The radiation observed from the rear hemisphere of the nozzle outlet is also computed in this work. Figure 12 shows that the radiation intensity observed from the rear hemisphere is plotted against the emission angle along the upright direction. Figure 13 shows that directed radiation has a maximal value at an emission angle of $70^{\circ}$, which is the deflection angle of $20^{\circ}$. By contrast, the directed radiation distribution under nondeflection condition has the maximum value at the right direction of $90^{\circ}$. Thus, AVEN remarkably changes the emission angle of the directed radiation. 


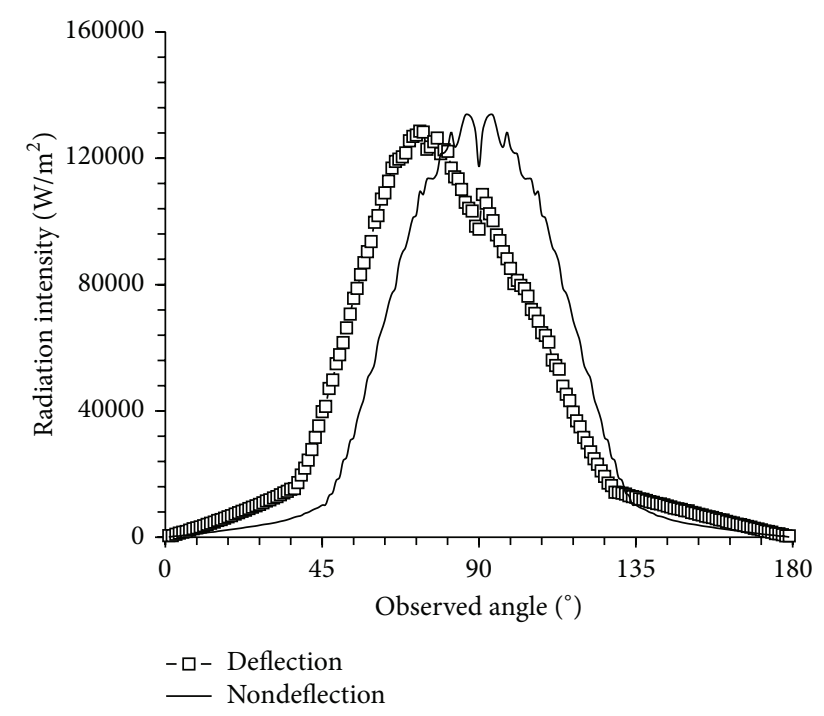

FIGURE 13: Radiation intensity in all wavebands on the outlet surface.

\section{Conclusion}

With the aim of designing the cooling structure design of the AVEN of a high-performance engine, this study established a heat transfer-spectral radiosity coupled heat balance equation model. A 3D all-structure nozzle simulation program of high accuracy was also developed by considering gas radiation heat transfer and film cooling. Our research obtained the following conclusions.

(1) The validation result of this work agrees well with the measured result in a reference, which demonstrates that this work may comprehensively reflect the heat transfer condition in the nozzle.

(2) The convergent part of the axial symmetric vectored nozzle studied in this work is shielded by the heat shield and cooling film. Thus, the wall temperature of this part is relatively low. By contrast, the wall temperature of the divergent part is high and should be the emphasis of the cooling design. The heat shield is the most severely heated structure of the nozzle, a phenomenon that must be considered by the design.

(3) The deflection of the axial symmetric vectored nozzle increases the wall temperature of the divergent part in the opposite direction of deflection and makes it last for a long distance at the downstream, which results in a severely heated area. The deflection also changes the distribution of the directed radiation at the outlet effectively.

\section{Conflict of Interests}

There is not conflict of interests for all authors of this paper.

\section{Acknowledgments}

This work is cosponsored by the National Natural Science Foundation of China (51376065, 51176052); Guangdong Key Scientific Project (2013B010405004); Guangdong Province Key Laboratory of Efficient and Clean Energy Utilization (2013A061401005), South China University of Technology; and Key Laboratory of Efficient and Clean Energy Utilization of Guangdong Higher Education Institutes (KLB10004).

\section{References}

[1] X.-Y. Zhang and X.-W. Wang, "Numerical research of infrared characteristics of axial-symmetrical vectored nozzle," Journal of Aerospace Power, vol. 19, no. 3, pp. 366-369, 2004.

[2] H. W. Deng, C. S. Zhao, D. B. Jia et al., "Analysis of stealthy shape design technology for aero-engine ex-haust nozzle," Aeroengine, vol. 40, no. 2, pp. 10-14, 37, 2013.

[3] W. S. Hines, J. R. McCarthy, and J. D. Seader, "Investigation of cooling problems at high chamber pressures," Tech. Rep. R3999(NASA CR-50773), Rocketdyne Division, North America Aviation, 1963.

[4] J. G. Lucas and R. L. Golladay, "Gaseous-film cooling of a rocket motor with injection near the throat," NASA TND-1988, 1988.

[5] M. E. Taslim, S. D. Spring, and B. P. Mehlman, "An experimental investigation of film cooling effectiveness for slots of various exit geometries," in Proceedings of the 26th AIAA/SAE/ASME/ASEE Joint Propulsion Conference, Orlando, Fla, USA, July 1990.

[6] C. Zhu and Z. Dong, "calculation of wall temperature of chambers with wigglestrip combustion film-cooled construction," Journal of Northwestern Polytechnical University, vol. 6, no. 1, pp. 53-61, 1988.

[7] Z. X. Han and G. J. Zhu, "Calculation of 2D wall tempera-ture distribution of film cooling combustion chamber flame tube," Journal of Aerospace Power, vol. 10, no. 1, pp. 83-86, 1995.

[8] B. G. Wang, Y. K. Li, and Z. Y. Hu, "Numerical analysis of the wall temperature with film-cooling in the combustion chamber," Acta Aeronautica et Astronautica Sinica, vol. 16, no. 4, pp. 415-421, 1995.

[9] H.-W. Zhang, W.-Q. Tao, Y.-L. He, and Z.-P. Feng, "Numerical study on coupled heat transfer of thrust chamber with regenerative cooling," Journal of Aerospace Power, vol. 21, no. 5, pp. 930-936, 2006.

[10] F. Han and Y. Liu, "Heat transfer characteristics of axi-symmetrical nozzle and RS nozzle," Journal of Aerospace Power, vol. 22, no. 11, pp. 1947-1953, 2007.

[11] Y.-H. Liu, J.-N. Li, and J. Cai, "Wall temperature calculation on an axi-symmtrical converging-diverging nozzle considering heat conduction, convection and radiation," Journal of Aerospace Power, vol. 23, no. 4, pp. 635-641, 2008.

[12] R. Duval, A. Soufiani, and J. Taine, "Coupled radiation and turbulent multiphase flow in an aluminised solid propellant rocket engine," Journal of Quantitative Spectroscopy and Radiative Transfer, vol. 84, no. 4, pp. 513-526, 2004.

[13] G. Nacouzi and D. Edwards, "The effects of radiation on film cooling in a Particle-Larden flow," in Proceedings of the National Heat Transfer Conference, San Diego, Calif, USA, August 1992.

[14] S. Y. Ge and D. Y. Liu, Film Cooling, Science Press, Beijing, China, 1985.

[15] R. Siegel and J. R. Howell, Thermal Radiation Heat Transfer, Taylor \& Francis, New York, NY, USA, 4th edition, 2002. 


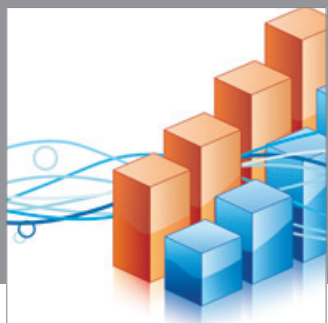

Advances in

Operations Research

mansans

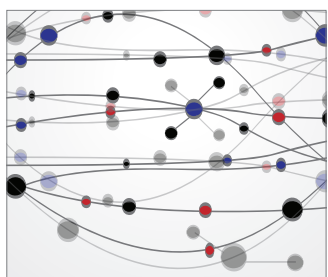

The Scientific World Journal
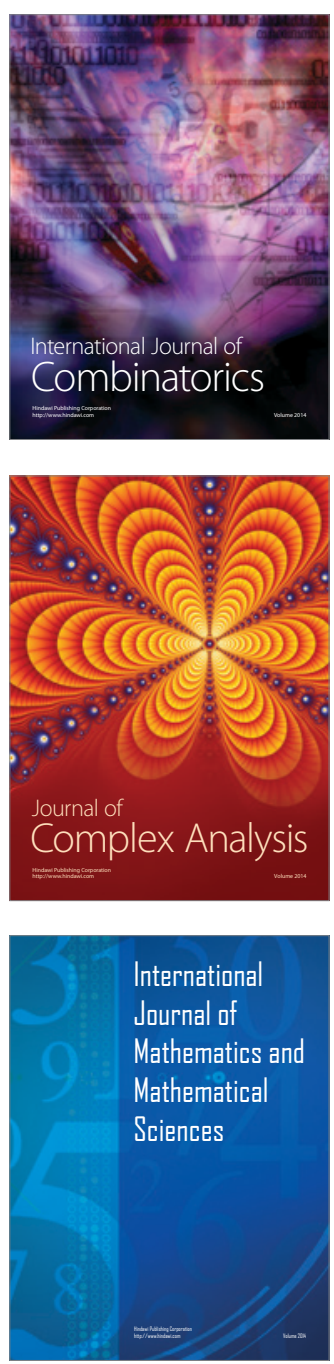
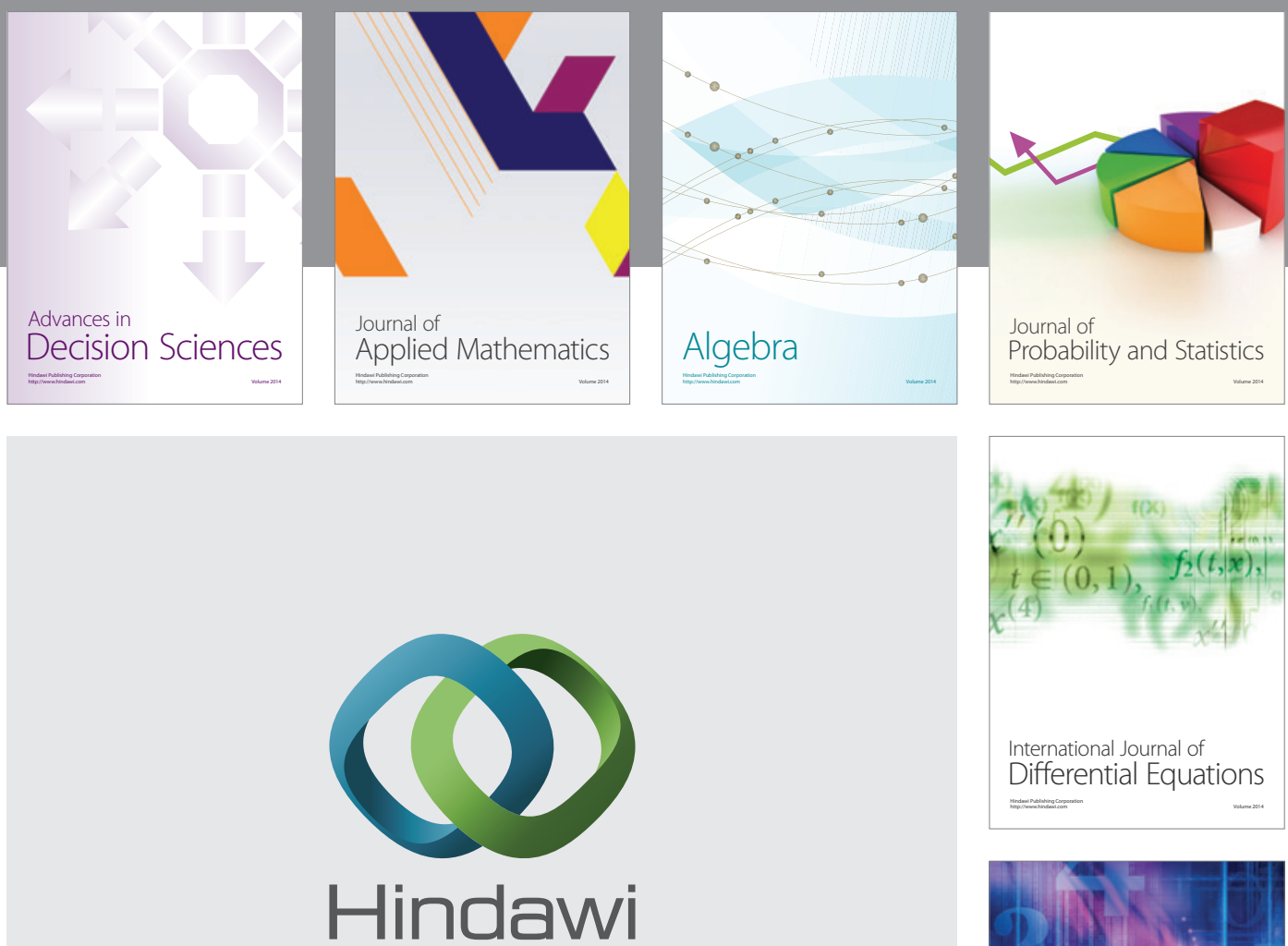

Submit your manuscripts at http://www.hindawi.com
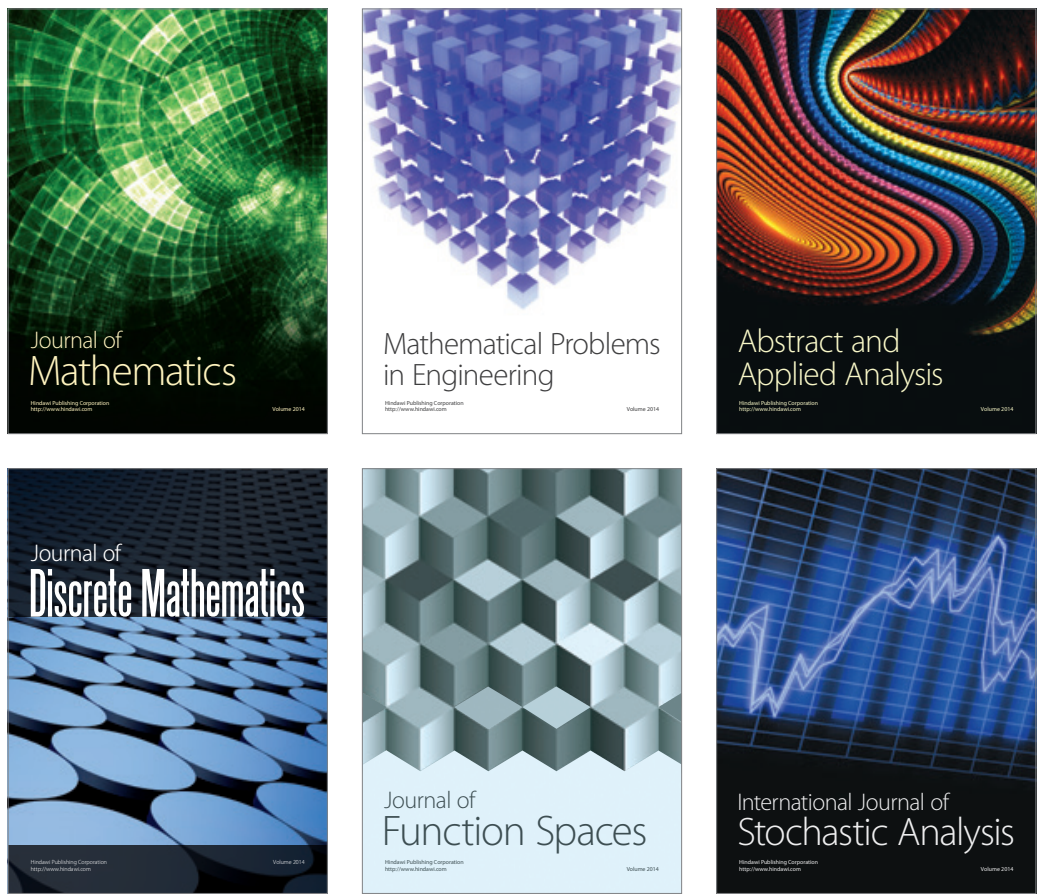

Journal of

Function Spaces

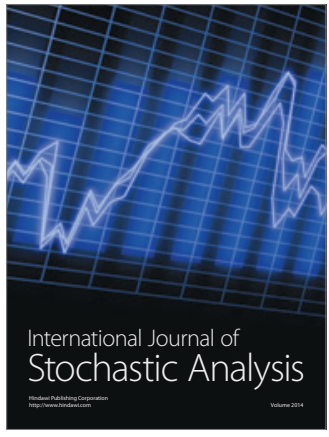

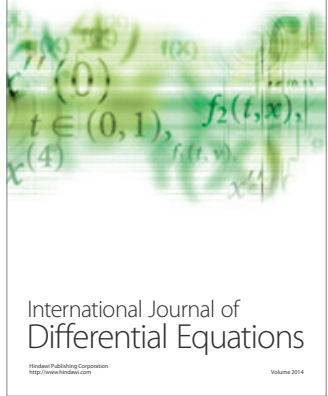
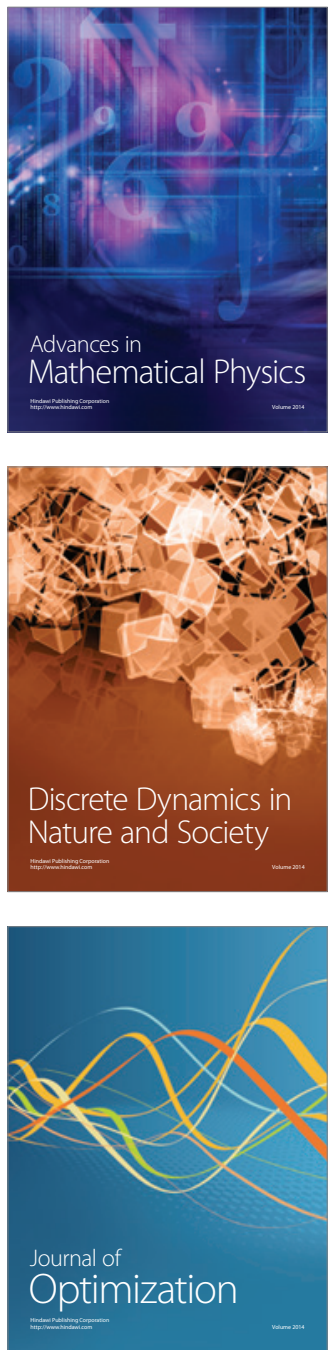